\title{
Predictive Targeted Movement in Electronic Spaces
}

\author{
Susanne Jul \\ University of Michigan \\ sjul@acm.org
}

\begin{abstract}
The lodestones and leylines interaction technique simplifies navigation in electronic spaces by coordinating physical and conceptual movement-gently constraining motion to follow automatically computed paths to predicted destinations. This approach simplifies physical movement, ensures that movement leads to interesting locations and supports navigation to locations not visible from the current location. It is illustrated in a spatial multiscale environment where pilot data show reliable performance improvements.
\end{abstract}

\section{KEYWORD}

Navigation, Locomotion, Steering, Movement, Multiscale, Zooming User Interface, Jazz, Lodestone, Leyline.

\section{INTRODUCTION}

A variety of techniques have been proposed for coordinating physical with conceptual movement in order to simplify navigation in electronic spaces. Approaches based on predicting likely destinations, e.g., using query relevance metrics [2] or history lists [8], ensure that movement leads to locations of interest. Approaches based on constraining movement, e.g., limiting movement to certain regions [3, 4], simplify physical movement. Approaches based on automatically computing paths of movement $[5,7]$ ensure that movement leads to locations of interest and simplify physical movement, but are limited to visible locations.

The lodestones and leylines technique introduced here combines these three approaches-destination prediction, constrained movement and automatically computed paths - in order to accomplish all three goals - simplify physical movement, ensure that movement leads to interesting locations and support navigation to locations not visible from the current location.

The technique limits conceptual movement to locationscalled lodestones (since they "attract" navigational attention) - that are relevant to the user's task or are necessary to navigation. Motion is constrained to follow leylines (named for lines of power in Celtic mythology) - paths that lead from the current location to a lodestone. Actual lodestones and leylines may be computed dynamically, but the designer determines what should qualify as a lodestone and how leylines should be computed. Lodestones typically correspond to conceptual elements of the user's task, e.g., files in a file navigation system or words in a documentediting task. Leylines are often direct paths, but could take obstacles (e.g., in a 3D environment) or other diversions into account. Physical movement is mapped onto the web of lodestones and leylines using a predictive utility (also defined by the designer) that uses user input to predict which lodestone is the intended target and select (or compute) the appropriate leyline to follow.

\section{LODESTONES AND LEYLINES IN JAZZ}

The lodestones and leylines technique has been applied to inter-object navigation in spatial multiscale worlds. Jazz [1, 6 ] is an application framework for designing and building multiscale electronic worlds. It uses an interaction metaphor of a conceptually infinite two-dimensional surface that can be viewed at an infinite range of magnifications. Objects have position and extent on the surface, and can appear differently, even becoming invisible, depending on the magnification (scale) of the view. Traditionally, movement is by panning (moving the view across the surface) and zooming (changing the scale of the view).

The prototype design assumes that the user's task is to move from one object to another, positioning each so that a reasonable amount of detail can be seen. Thus, lodestones are, with one exception, views of individual objects and leylines are direct paths through space and scale. Prediction of target destinations is based on geometric distance potential targets and the mouse.

In order to initiate movement, the user indicates the desired direction of zoom (in or out) by pressing the appropriate mouse-button (Figure 1:1). If zooming in, the system uses the mouse location to select the nearest lodestone (regardless of whether it is visible) as the predicted destination and computes and begins to zoom along the leyline that will center this lodestone in the view (Figure 1:1-3). Moving the mouse to be closer to a different lodestone during zoom-in changes the target prediction (Figure 1:2',2). The system immediately detects the new destination and computes the new leyline to be followed. This allows easy error correction and scanning of potential destinations. If no lodestone can be reached by zooming in, zoom-in is not permitted.

If zooming out, the destination is assumed to be a special lodestone, the Top of the World view (Figure 1:1). This is the most magnified view that contains all lodestones in the world. Zoom-out is not permitted past this point, since all lodestones are already within reach of the mouse. Stopping zoom-in and zoom-out when no more lodestones can be reached prevents users from getting lost in empty space (a 


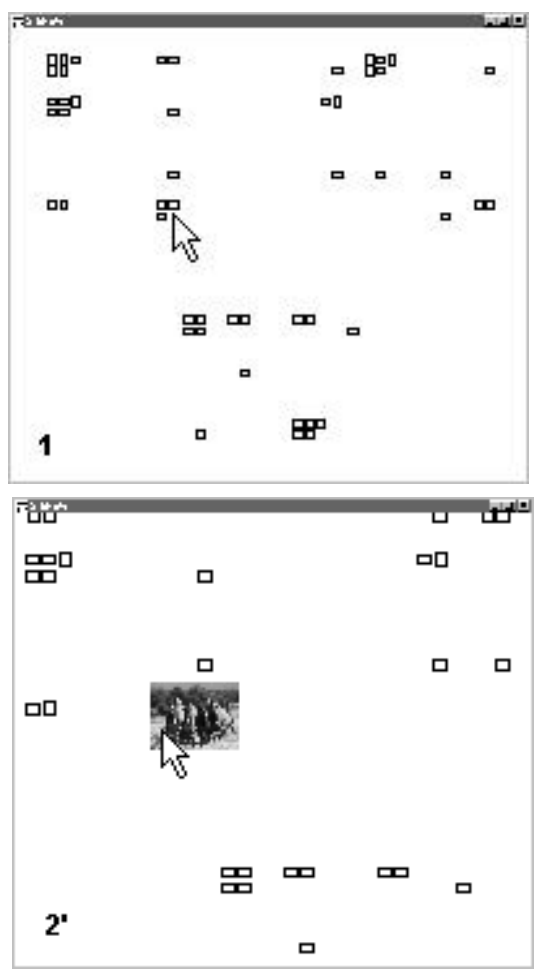

significant danger in spatial multiscale environments). The Top of the World lodestone provides users a sure means of recovery from disorientation in occupied space.

In a simple comparison, lodestones and leylines ("Leylines") was reliably faster than traditional ("Pad++") movement ( $\mathrm{p}<.05$, Figure 2). Subjects appeared to require much less "stop and go" movement, and a majority spontaneously reported feeling less lost and more confident.

\section{SUMMARY}

The lodestones and leylines technique combines destination prediction, constrained movement and automatically computed paths to support navigation in electronic spaces. The example design presented employs simple definitions of lodestones and leylines, and a simple utility for target prediction. However, the technique can readily accommodate more complex definitions, e.g., allowing collections of objects to be lodestones, requiring leylines to move through
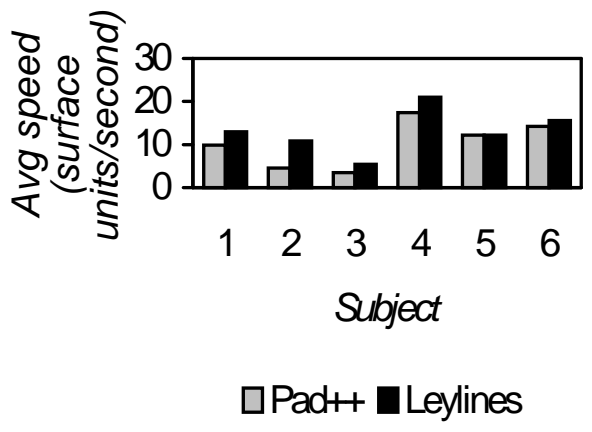

Figure 2 Average speed of movement for two movement techniques, paired by subject. $t=3.02(p<.05)$. nested groups and basing prediction on a relevance factor such as recency of use or semantic salience. A significant benefit of predictive targeted movement is to provide safe actions for users to take if they become lost or disoriented.

\section{REFERENCES}

1. Bederson, B., Meyer, J., Good, L. (2000). Jazz: An Extensible Zoomable User Interface Graphics Toolkit in Java. Proceedings of ACM UIST 2000, ACM Press.

2. Frants, V. J., Shapiro, J., and Voiskunskii, V. G. (1997). Automated Information Retrieval: Theory and Methods. San Diego: Academic Press.

3. Galyean, T. A. (1995). Guided Navigation of Virtual Environments. 1995 Symposium on Interactive 3D Graphics. ACM Press. 103-104.

4. Hanson, A. J., Wernert, E. A., Hughes, S. B. (1999). Constrained Navigation Environments. In Hagen, H., Nielson, G. M., and Post, F. (eds.), Scientific Visualization: Dagstuhl '97 Proceedings, IEEE Computer Society Press. 95-104.

5. Igarashi, T., Kadobayashi, R., Mase, K., Tanaka, H. (1998). Path Drawing for 3D Walkthrough. Proceedings of ACM UIST 98, ACM Press, 173-174.

6. http://www.cs.umd.edu/hcil/jazz

7. Mackinlay, J. D., Card, S. K., Robertson, G. G. (1990). Rapid Controlled Movement Through a Virtual 3D Workspace. SIGGRAPH '90 Conference Proceedings, in Computer Graphics 24 (4), 171-176.

8. Nielsen, J. (1990). The Art of Navigating Through Hypertext. Comm. ACM 33, 3 (Mar.), 296-310. 